\title{
Méthode de tracé des écoulements réels à faibles pertes d'énergie
} (culottes, convergents, bâches, etc.)

\author{
Method of obtaining actual flow patterns \\ with low energy loss
}

("Y" branchs, convergent sections, spiral casings, etc.)

\author{
PAR R. MEYER \\ INGÉNIEUR AUX ÉTABLISSEMEN'TS NEXTPIC \\ PROFESSEUR A L'E.N.S.E.H.G.
}

\begin{abstract}
Les tracés d'écoulements irrotationnels de fluide parfait (surtout depuis la mise au point des méthodes de MM. Barrillon, Danel, Sauvage DE SaINT-Marc, etc.) ont forgé une base d' "intuition》des formes hydrauliques chez lons les ingénieurs hydrauliciens. En fait, les écoulements réels sont en général assez différents des écoulements irrotationnels. Dans cette communication:

$1^{\circ}$ On montre que beaucoup d'écoulements réels importants pour l'industrie sont peu différents d'écoulements rotationnels de fluide parfait; $2^{\circ}$ On expose une méthode de tracé graphique des écoulements rotationnels de fluide parfait qui sont à deux dimensions ou qui peuvent être ramenés à deux dimensions;

$3^{\circ}$ On indique quelques propriétés intéressantes, déduites de ces tracés.
\end{abstract}

\begin{abstract}
Irrotational flow patterns of a perfect fluid (particalarly since the development of the method of MM. Barillon, Danel, Sauvage DE SaINT-Maric, etc.) have formed an "intuitive" basis for the hydranlic forms used by all hydraulic engineers. In fact, actual flows are, in general, quite different from irrotational flows. In this article:

1. It is shown that many actual flows encountered in industry are little different to rotational flows of a perfect fuid.

2. It describes a method of graphically lruwing perfect fluid rotational flows which are either two dimensional or which can be rendered two dimensionnally.

3. It describes certuin interesting deductions which can be made from these drauings.
\end{abstract}

\section{I. - INTRODUCTION. - BUT DE LA PRESENTE COMMUNICATION}

Le but de la présente communication est d'étudier un certain nombre d'écoulements, tels que les écoulements dans les culottes, les bâches des turbines, les convergents et divergents, etc.

Pendant très longtemps, les chercheurs ont essayé d'étudier ces écoulements en faisant des hypothèses simplificatrices telles que l'irrotationnalité de ces écoulements. En fait, les écoulements réels restaient toujours fort éloignés des écoulements fictifs ainsi obtenus. Une hypothèse relativement récente consiste à admettre que ces écoulements sont proches d'écoulements rotationnels de fluide parfait. Il se trouve que cette hypothèse conduit à des écoulements dont la différence avec les écoulements réels est très faible, comme le témoignent de nombreux essais faits jusqu'ici. Néanmoins, cette théorie n'a pas encore été très développée, d'une part parce qu'elle est relativement récente et d'autre part parce qu'elle mène à des problèmes mathémati- 
ques très compliqués. Dans la présente communication, nous allons d'abord rappeler les bases physiques sur lesquelles se fonde cette théorie (encore relativement peu connue); ensuite nous allons montrer comment on peut obtenir graphiquement de tels écoulements et finalement nous allons indiquer quelques propriétés nouvelles de ces écoulements.

Pour fixer les idées, nous donnons de suite les limites d'application de la méthode de tracé graphique que nous exposerons. Par la suite, nous reviendrons plus amplement sur chacune des limitations mentionnées ci-dessous. Ces limitations sont les suivantes :

1. La méthode ne s'applique que si les phéno- mènes laminaires et de turbulence naissante sont négligeables dans la partie de l'écoulement à étudier.

2. Elle ne s'applique qu'aux parties d'écoulement dans lesquelles les pertes d'énergie sont relativement faibles.

3. Comme toutes les méthodes graphiques (et même semi-graphiques), elle ne s'applique qu'aux écoulements à deux dimensions, ou ceux que l'on peut ramener à deux dimensions d'une façon ou d'une autre.

4. Elle ne s'applique qu'aux écoulements permanents (ou permanents en moyenne au sens que l'on donne à ce terme en turbulence).

\section{II. - RAPPELS SUR LA PHYSIQUE DES ECOULEMENTS DONT IL EST QUESTION DANS CETTE COMMUNICATION}

La première théorie qui a servi à étudier les écoulements définis plus haut a été celle dite « des fluides parfaits », c'est-à-dire des fluides de viscosité rigoureusement nulle.

Cette théorie donne entre autres les résutats suivants :

1. Il se trouve qu'il est impossibble de tenir compte du phénomène de l'adhérence des fluides aux parois solides qu'ils touchent.

Le phénomène de l'adhérence a été constaté expérimentalement pour tous les fluides. Néanmoins, la théorie des fluides parfaits ne permet pas d'en tenir compte : la condition d'adhérence est incompatible avec les propriétés des écoulements des fluides parfaits. Il n'y a pas lieu de s'étonner de ce fait : il existe dans toute théorie simplifiée des contradictions avec la réalité, et si de telles contradictions n'existaient pas, la théorie ne serait pas une théorie simplifiée, mais la théorie exacte.

2. La théorie des fluides parfaits montre que tout écoulement qui a lieu dans le champ de la gravitation terrestre est forcément irrotationnel.

Cette théorie permet en pratique d'étudier les écoulements réels qui sont peu rotationnels. En fait, si on mesure les vitesses dans les écoulements réels, on s'aperçoit que la plupart sont fortement rotationnels, soit localement, soit totalement.

La deuxième théorie ayant servi à étudier les écoulements définis ci-dessus a été celle des fluides à viscosité relativement faible, c'est-à-dire la théorie des écoulements à nombre de Reynolds élevé.
Dès que la viscosité n'est pas supposée être nulle, même si elle est extrêmement faible, des faits nouveaux et très importants apparaissent dans la théorie. Parmi ces faits, il y a :

1. La possibilité et la nécessité de tenir compte de l'adhérence des fluides aux parois solides.

Tant que le fluide est loin des parois, l'écoulement est voisin de l'écoulement irrotationnel de fluide parfait. Dès que le fluide s'approche d'une paroi (ou d'une interface entre deux fluides), il y a formation d'une couche limite près de cette paroi (théorie de Prandtl). Dans cette couche limite, l'écoulement du fluide réel diffère forlement de l'écoulement irrotationnel du fluide parfait, puisque l'un adhère à la paroi et l'autre ne peut pas adhérer. L'épaisseur de cette couche croît au fur et à mesure que le fluide longe la paroi.

C'est ce phénomène qui produit les tourbillons existant dans les écoulements réels.

2. La viscosité détruit les tourbillons qui se trouvent au sein du fluide et limite par là le rotationnel.

Cette théorie semble pouvoir s'appliquer dans presque tous les cas pratiques. En fait, les couches limites deviennent rapidement turbulentes et de toute façon cette théorie mène à des problèmes que les connaissances et les moyens actuels des mathématiques ne permettent pas de résoudre, sauf dans quelques cas simples.

Aussi les praticiens ont-ils essayé depuis lonstemps de trouver une théorie à la fois plus exacte que celle des écoulements irrotationnels des fluides parfaits et moins difficile à mettre en ouvre 
que la théorie des fluides visqueux. C'est ainsi qu'ont été élaborées les différentes théories d'écoulements plus ou moins rotationnels de fluides parfaits, c'est-à-dire d'écoulements rigoureusement impossibles dans le champ de gravitation terrestre si le fluide était réellement parfait, sauf ceux existant depuis «l'origine des temps》.

Parmi ces théories, il y a en particulier celle des tourbillons 《liés 》 aux obstacles entièrement immergés dans le fluide et celle des écoulements irrotationnels contenant des parties d'écoulement rotationnelles (théorie des ailes d'envergure limitée de Prandtl, théorie des files de tourbillon de Bénard et Von Karman, etc.). Néanmoins, dans toutes ces théories on part essentiellement d'une partie d'écoulement qui est irrotationnelle.

Dans de nombreux écoulements, l'intensité du rotationnel est du même ordre de grandeur partout; ces écoulements ne peuvent pas être étudiés avec les théories ci-dessus. Il faut dans ce cas employer la théorie des écoulements rotationnels des fluides parfaits. Cette dernière théorie englobe toutes les autres théories d'écoulements des fluides parfaits qui ne sont que des cas particuliers de cette théorie générale (cas particuliers plus simples à mettre en œuvre dans certains cas).

Jusqu'à présent, la théorie des écoulements rotationnels des fluides parfaits n'a fait l'objet que d'un nombre assez petit d'études et de publications. En effet, cette théorie mène à des équations mathématiques relativement compli- quées. Par contre, les résultats expérimentaux obtenus jusqu'à présent, et dont nous parlerons plus loin, montrent que cette théorie permet effectivement d'étudier avec une approximation très suffisante un grand nombre d'écoulements que l'on rencontre dans la pratique et que l'on n'a pas pu étudier de façon satisfaisante avec les théories antérieures.

Le but de cette communication est d'abord d'exposer une méthode de tracé graphique du type 《méthode de Lehmann » pour les écoulements rotationnels des fluides parfaits et ensuite de montrer qu'on peut en tirer une certaine vue d'ensemble sur les propriétés des écoulements cherchés.

En effet, jusqu'à présent, seul un nombre assez restreint d'écoulements rotationnels de fluide parfait ont été obtenus et il n'existe aucune synthèse relative à ces écoulements. On ne peut donc guère se faire une idée à priori d'un tel écoulement, donc essayer d'orienter une recherche de formes hydrauliques pour une culotte, une bâche, un divergent, etc.

Une méthode graphique n'intéressera probablement que peu les théoriciens de la mécanique des fluides et les mathématiciens (qui autrement l'auraient certainement trouvée depuis longtemps); par contre, elle pourra servir aux ingénieurs pour qui seuls les résultats pratiques comptent et qui préfèrent souvent les graphiques qui sont plus «parlants » aux calculs qui sont (surtout dans le cas actuel) d'une complexité théorique assez grande.

\section{III. - BASES PHYSIQUES DE LA THEORIE DES ECOULEMENTS ROTATIONNELS DES FLUIDES PARFAITS}

Rappelons que dans un écoulement de fluide parfait, même rotationnel, les tourbillons se conservent indéfiniment pareils à eux-mêmes. Un tel écoulement ne saurait donc servir à étudier un écoulement réel dans lequel il y a une production ou une dissipation importante de tourbillons. Nous allons néanmoins montrer comment la théorie des écoulements rotationnels des fluides parfaits peut servir à étudier des problèmes pratiques avec une très bonne approximation. Pour simplifier l'exposé, nous envisagerons un cas particulier, qui est, par ailleurs, un cas important dans la pratique : cclui des culotles.

Sur la figure 1 se trouve un écoulement comprenant une culotte. Dans la partie « $A »$ de cet écoulement, l'eau sort d'un grand réservoir et

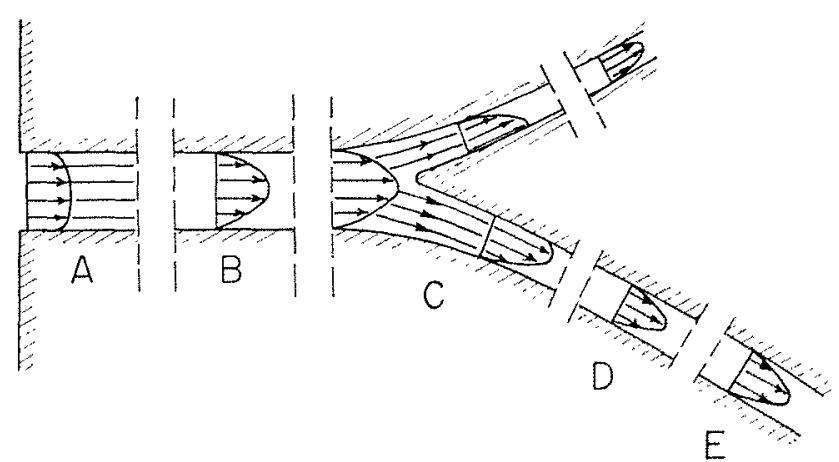

Fig. 1

entre dans une conduite. La courbe des vitesses à l'entrée de la conduite est très schématique- 
ment indiquée sur la figure 1. Dans cette partie, le liquide se comporte pratiquement comme s'il était parfait. Dans la partie «B $\gg$, l'influence des parois se fait de plus en plus sentir et la courbe des vitesses tend vers son profil d'équilibre bien connu. L'ćcoulement est irrotationnel à l'entrée de la partie « $\mathrm{B} »$, mais est fortement rotationnel à la sortie. Dans cette partie de l'écoulement, l'influence de la viscosité n'est évidemment pas négligeable et le fluide ne peut pas être considéré comme parfait, même en première approximation.

Dans la partie « $\mathrm{C} »$, il y a un grand changement dans les formes hydrauliques sur une longueur relativement courte; dans cette partie de l'écoulement, la production et la destruction des tourbillons sont négligeables si toutefois la culotte est correctement construite. Nous reviendrons d'ailleurs bientôt sur le fait que la culotte doit être correctement construite. Dans la partie «D D, un nouvel état d'équilibre se développe; il y a donc à nouveau production et destruction non négligeables de tourbillons. Enfin, dans la partie «E», le nouvel état d'équilibre est atteint.

En résumé, seule dans la partie « $\mathrm{C} »$, la viscosité peut jouer un rôle négligeable et ce n'est que dans cette partie que la théorie des écoulements rotationnels de fluides parfaits a des chances de pouvoir s'appliquer. Pour savoir si cette théorie s'applique effectivement, on ne peut évidemment se baser que sur des essais.

Les essais justificatifs de cette théorie qui ont été faits jusqu'à présent et dont nous parlerons plus loin permettent effectivement d'affirmer que cette théorie s'applique aux culottes.

On peut se demander si la théorie des écoulements irrotationnels des fluides parfaits ne s'appliquerait pas également aux culottes avec une approximation suffisante. La différence entre les deux théories réside essentiellement dans la forme de la courbe des vitesses à l'entrée de la culotte, c'est-à-dire de la partie « $\mathrm{C}$ » de l'écoulement de la figure 1. Si on emploie la théorie des écoulements rotationnels on peut se donner la courbe de répartition exacte des vitesses à l'entrée de la culotte. Les erreurs que l'on fera seront dues à l'influence de la viscosité dans la culotte; on obtient un écoulement exact à l'entrée de la culotte. Cet écoulement s'éloignerait progressivement de l'écoulement réel si la culotte était très longue. Si on emploie la théorie des écoulements irrotationnels, on est par définition obligé de se donner à l'entrée de la culotte une courbe de répartition des vitesses ne contenant pas de rotationnel, c'est-à-dire une courbe qui n'a qu'une relation très vague avec la courbe réelle : seul le débit total peut par exemple être conservé. L'écoulement oblenu n'est donc nulle part voisin de l'écoulement réel.
Nous avons admis jusqu'à présent que la culotte était correctement tracée, e'est-à-dire qu'il n'y' avait pas de décollement à l'intérieur. Un décollement provoque une forte production de tourbillons et il faut voir dans quelle mesure la théorie des écoulements rotationnels des fluides parfaits reste applicable. En pratique, un décollement produit une « surface de décollement $\gg$ dont on peut tenir compte dans une certaine mesure, comme on le fait dans la théorie des lignes de jet ou de sillage des écoulements irrotationnels des fluides parfaits. Au fur et à mesure que la surface de décollement s'étend à l'aval des points de décollement, elle devient de moins en moins nette dans l'écoulement réel et finit par cesser d'exister : les tourbillons nouvellement créés par le décollement envahissent toute la section de l'écoulement. La théorie des écoulements rotationnels des fluides parfaits avec surface de sillage s'applique donc à l'amont du décollement et à une distance égale à deux ou trois fois le diamètre de l'écoulement à l'aval du décollement.

En pratique, le problème hydraulique posé par les culottes est précisément de déterminer les formes hydrauliques à donner à la culotte pour éviter la formation d'un décollement qui entrâ̂nerait une forte perte de charge. En général, ce décollement doit être évité pour différents modes de répartition du débit entre les deux branches de la culotte. Enfin, on a remarqué que les culottes bien tracées du point de vue hydraulique avaient des formes souvent voisines des meilleures formes quant à la résistance du matériau de la culotte. Ceci peut d'ailleurs s'expliquer dans une certaine mesure.

Ajoutons quelques mots sur les pertes de charge des culottes pour montrer comment la théorie des écoulements rotationnels peut permettre de concevoir ce problème de façon spécialement claire. Dans le cas des culottes sans décollement, on peut mesurer sur une installation du type figure 1 une certaine perte d'énergie entre l'entrée de la culotte et la sortie de la partie «D D de l'écoulement. Cette perte d'énergie provient de deux phénomènes:

1. La perte normale dans le tronçon 《 $\mathrm{D} \gg$;

2. Le changement dans la forme de la courbe de répartition des vitesses entre l'entrée et la sortie de $\ll D$ D.

Seul le dernier phénomène est imputable à la culotte et varie si on change de: culotte. On pourrait donc penser que ce phénomène permet de définir la perte de charge de la culotte. En réalité, les deux phénomènes ci-dessus ne sont nullement additifs. En plus, la répartition des vitesses à la sortie de la culotte ne dépend pas seulement de la culotte mais aussi de la courbe de répartition des vitesses à l'entrée de la culotte. 
On en déduit qu'il est impossible de définir la perte de charge d'une bonne culotte; seule la perte d'énergie de l'ensemble des parties «B», 《 $\mathrm{C} \gg$ et $\ll \mathrm{D} »$ de la figure 1 peut être définie correctement. Par contre, si la culotte est mauvaise et présente un décollement (et ceci est malheureusement le cas de beaucoup de culottes existantes), ce décollement provoque une perte d'énergie très importante par rapport aux phénomènes décrits plus haut et on peut très souvent définir avec une approximation suffisante la perte de charge d'une telle culotle; en tout cas elle ne dépend que peu de la partie «D». On peut dans ce cas aussi la calculer en première approximation à l'aide de la théorie des écoulements rotationnels et de la formule des élargissements brusques. Dans le cas des bonnes culottes, il faut faire des essais, extrêmement délicats d'ailleurs, la perte d'énergie étant parfois extrêmement petite. Néanmoins, il semble qu'elle soit toujours positive.

Une constatation importante mise en évidence par l'exemple ci-dessus est que la théorie des écoulements rotationnels des fluides parfaits ne peut servir qu'à l'étude d'une ou plusieurs parties d'un écoulement, jamais d'un écoulement entier. En effet, si on détermine un écoulement de fluide parfait entier, cet écoulement serait forcément irrotationnel, aucun tourbillon ne pouvant naître dans un tel ćcoulement. Par contre, si on admet que la viscosité a un effet non négligeable dans une première partie d'un écoulement, des tourbillons y naissent. Si on peut admetlre (comme dans le cas des culottes) que dans une deuxic̀me partie de cet écoulement le fluide est parfait, l'écoulement y sera néanmoins rotationnel : les tourbillons créés dans la première partie traverseront la deuxième.

On voit par là que les écoulements rotationnels des fluides parfaits ne sauraient probable- ment pas être appliqués en aérodynamique, où il est impossible de découper les écoulements en «parties » à la sortie desquelles on connaît le rotationnel. Par contre, en hydraulique et en particulier en hydroélectricité, il existe souvent des écoulements comprenant des conduites ou des canaux suivis de changements assez importants des formes hydrauliques sur un court parcours. A la sortie de la conduite ou du canal, on connaît souvent la répartition des vitesses, donc des tourbillons. Les changements rapides de formes hydrauliques peuvent être des culottes, des convergents, des divergents, des coudes, des machines hydrauliques, etc.

Mentionnons maintenant quelques études expérimentales qui ont eu pour but de démontrer la validité de la théorie des écoulements rotationnels des fluides parfaits dans les cas indiqués cidessus :

STRSChELETzKy : Strömung im Uebergangsraum der Wasserturbinen - Ingenienr-Archiv, vol. 19 (1951), pp. 309-320.

Rectificatif, Ing.-Archil. vol 21 (1953), p. 408.

Eichenberger : Secondary flow within a bend Journal of Mathematics and Physics, vol. 32 (1953), pl. $39-42$.

Hawthorne: Secondary circulation in fluid flow. M.I.T. Gas lurbine Laboratory.

A theory of shuts in Boundary Layers. M.I.T. Gas turbine Laboratory.

Signalons que, dans certains cas, les auteurs ont également montré la différence entre les écoulements rotationnels ef irrotationnels, ces derniers donnant en général des résultals fort éloignés des résultats expérimentaux.

\section{IV. - CONSIDÉRATIONS GÉNERALES SUR LES TRACÉS GRAPHIQUES D'ÉCOULEMENT. ÉCOULEMENTS PRESQUE PLANS}

Comme nous l'avons signalé au début de cette communication, la méthode qui sera exposée ici ne s'applique (comme toutes les méthodes graphiques) qu'aux écoulements à deux dimensions et à ceux que l'on peut y' ramener.

En effet, dans tous les écoulements de fluide, le mode d'écoulement en un point est influencé par le mode d'écoulement en tous les autres points de cet écoulement. On ne peut done pas étudier un écoulement par «tranches » indépendantes, sauf si une tranche contient tous les renseignements sur l'écoulement, c'est-à-dire si l'écoulement est à deux dimensions ou peut s'y ramener. Par ailleurs, on peut étudier graphiquement, dans ce cas, tous les écoulements. Dans la présente communication nous parlerons des écoulements suivants (les plus importants parmi ceux qui sont à deux dimensions ou qui peuvent s'y ramener) :

1. Les écoulements plans; 
2. Les écoulements que nous appellerons «presque plans » et que nous définirons ci-dessous;

3. Les écoulements de révolution (méridiens ou non).

En pratique, aucun écoulement n'est plan. En effet, il faudrait que les vitesses soient les mêmes le long de chaque perpendiculaire au plan de l'écoulement. Or les écoulements pratiques sont toujours limités par des parois et la vitesse décrôt, si on se rapproche d'une paroi.

Par contre; de nombreux écoulements sont «presque plans » (*). Pour définir ces écoulements, revenons aux écoulements plans théoriques. Ces écoulements ne sont pas forcément limités par deux plans parallèles. Les parois peuvent aussi être constituées par une famille de lignes de courant non coplanaires. Chaque ligne de courant doit être une courbe plane, mais on peut associer des lignes de courant appartenant à des plans différents et former ainsi une paroi courbe. Inversement, un écoulement plan ne peut être limité que par une paroi dont les intersec-
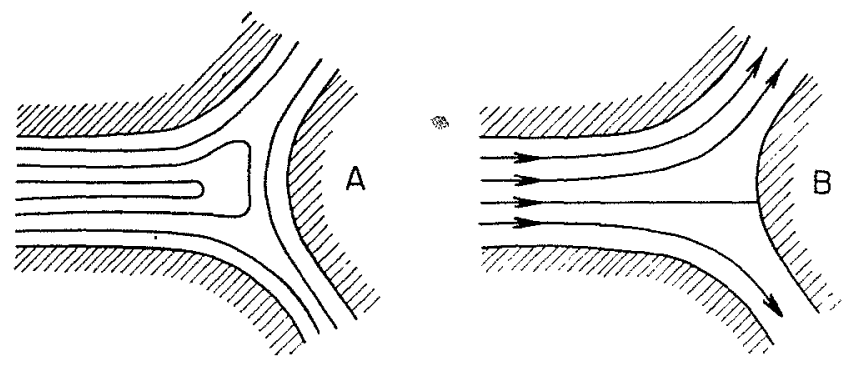

Fig. 2 tions avec les plans parallèles au plan de l'écoulement sont des lignes de courant et se projette donc orthogonalement sur ce plan suivant des lignes de courant. En effet, chaque particule suit une trajectoire et reste donc dans un plan, en particulier une particule prise sur une paroi. Mais une particule située sur la paroi le reste par ailleurs indéfiniment.

Dans de nombreux écoulements, les intersections des parois avec des plans parallèles se projettent sur l'un d'entre eux suivant des courbes voisines des trajectoires de ce plan (cf. fig. 2 par exemple). Dans ce cas, nous dirons que l'écoulement est presque plan et peut être étudié dans un plan moyen en affectant à chaque point de ce plan la valeur moyenne de chaque variable sur une perpendiculaire à ce plan. Ainsi on considérera la vitesse moyenne sur une perpendiculaire et non la vitesse locale. Cette idée n'est pas nouvelle; elle sert en particulier quand on étudie les écoulements irrotationnels par l'analogie dite de «Stokes».

L'écoulement réel est d'autant plus éloigné de l'écoulement presque plan que la hauteur de l'écoulement varie plus et plus rapidement le long d'une trajectoire; les variations de hauteurs perpendiculaires aux trajectoires ne sont pas gênantes. En outre, les courants «secondaires » (phénomènes à trois dimensions, donc non représentables graphiquement) sont des sources de perturbations. Néanmoins, leur intensité n'intervient pas directement, mais seulement leur interaction avec le courant moyen. Cette intersection n'est souvent de loin pas aussi grande qu'on pourrait le craindre a priori.

\section{V. - PROPRIETES DES ECOULEMENTS ROT ATIONNELS DES FLUIDES PARFAITS SERVANT AUX TRACÉS GRAPHIQUES}

Pour établir ces propriétés, il faut évidemment partir des équations régissant les écoulements correspondants. On peut passer ce paragraphe sans que cela nuise à la compréhension du reste.

Les équations de départ sont :

$$
\overrightarrow{\mathrm{V}}_{\mathrm{A}} \overrightarrow{\operatorname{rot}} \overrightarrow{\mathrm{V}}=-\overrightarrow{\operatorname{grad}}\left(\frac{p}{\rho}+\mathrm{U}+\frac{\mathrm{V}^{2}}{2}\right)
$$

(*) Les écoulements 《presque plans » irrotationnels ont été définis et étudiés il y a longtemps par ii. DanEL, président de la SO.G.R.E.A.H., dans une étude non publiée.

$$
\begin{aligned}
& \mathrm{V}=\text { vecteur vitesse, } \\
& \mathrm{U}=\text { fonction des forces extérieures, } \\
& p=\text { pression, } \\
& \mathrm{\rho}=\text { masse spécifique. }
\end{aligned}
$$

Posons $p / \bar{\omega}+\mathrm{U} / g+\mathrm{V}^{2} / 2 g=\mathrm{H}$. Nous appellerons $\mathrm{H}$ la charge; $g=9,81 \mathrm{~m} / \mathrm{s}^{2}$ : accélération de la pesanteur.

De (1) on déduit par multiplication scalaire par $\vec{V}$ :

$$
\overrightarrow{\mathrm{V}} \cdot \overrightarrow{\mathrm{grad}} \mathrm{H}=0
$$


Nous appellerons les surfaces $\mathrm{H}=$ Cste : surfaces équicharge.

L'équation (2) montre que les lignes de courant sont des lignes tracées sur les surfaces équicharge.

Considérons maintenant plus particulièrement les écoulements presque plans (dont les écoulements plans sont un cas particulier). On peut alors graduer les lignes de courant en fonction du débit $Q$ passant entre chacune d'entre elles et une ligne prise comme référence. De l'équation (2) on tire alors $\mathrm{H}=\mathrm{H}(\mathrm{Q})$. Par symétrie, le vecteur rot $\vec{V}$ est perpendiculaire au plan de l'écoulement, donc à $\vec{V} \cdot \vec{V}_{A}$ rot $\vec{V}$ est donc dans le plan de l'écoulement et on a :

$$
/ \mathrm{V} / / \operatorname{rot} \mathrm{V} /=/ \operatorname{grad} \mathrm{H} /
$$

\section{/ / : valeur absolue.}

On peut repérer les points du plan par la valeur $Q$ de la ligne de courant qui $y$ passe et par une deuxième coordonnée $\| x »$ quelconque. On déduit alors de (3) :

D'où :

$$
\frac{d \mathrm{Q}}{d x} / \operatorname{rot} \mathrm{V} /=\frac{d \mathrm{H}(\mathrm{Q})}{d x}
$$

$$
/ \mathrm{rol} \mathrm{V} /=f(\mathrm{Q})
$$

Le rotationnel se conserve donc le long des lignes de courant. Cette propriété suffira pour obtenir la relation de base de la méthode graphique.

En observant que le vecteur $\vec{V}$ a pour composantes :

$$
\begin{gathered}
\frac{\partial Q}{\partial y} ;-\frac{\partial Q}{\partial x} \quad \text { et que } \overrightarrow{\operatorname{rot}} \vec{V}=\Delta Q \\
\left(\Delta=\frac{\partial^{2}}{\partial x^{2}}+\frac{\partial^{2}}{\partial y^{2}}\right)
\end{gathered}
$$

on obtient, en prenant le rotationuel de (1):

$$
\frac{\partial \Delta Q}{\partial x} \quad \frac{\partial Q}{\partial y}=\frac{\partial \Delta Q}{\partial y} \quad \frac{\partial Q}{\partial x}
$$

D'où l'on peut aussi tirer :

$$
/ \operatorname{rot} \mathrm{V} /=\Delta \mathrm{Q}=f(\mathrm{Q}) \text {. }
$$

On démontre de la même façon les propriétés suivantes des écoulements de révolution :

1. Par symétrie les lignes de courants sont tracées sur des surfaces cerclées que nous appellerons : surface de courant. On peut les graduer en affectant à chacune d'entre elles le débit total qui passe à l'intérieur : $Q$;

2. On démontre que les surfaces équicharge et les surfaces de courant sont confondues : $\mathrm{H}=\mathrm{H}(\mathbf{Q})$;

3. On démontre que la composante $《 v »$ de la vitesse le long des cercles parallèles est de la forme $[k(Q)] / r: r$ distance du point considéré à l'axe de révolution. Sur chaque surface de courant, l'écoulement se fait selon une loi de vortex;

4. La composante du rotationnel le long des cercles parallèles est de la forme :

$$
\frac{k(\mathrm{Q}) \cdot k^{\prime}(\mathrm{Q})}{r}+r \cdot g(\mathrm{Q})
$$

Ces propriétés suffisent pour établir la méthode du tracé graphique.

Dans le cas des écoulements presque plans, on trouve :

$$
/ \operatorname{rot} \mathrm{V} /=\mathscr{H} \cdot f(\mathrm{Q})
$$

où He est la hauteur locale de l'écoulement.

\section{VI. - DESCRIPTION DE LA METHODE DE TRACE GRAPHIQUE. - EXEMPLES}

Pour déterminer les écoulements, on cherche à tracer" les lignes de courant. En outre, l'application de la méthode ci-contre exige le tracé du faisceau des courbes orthogonales au faisceau des lignes de courant. On trace effectivement toutes les lignes de cote $k \delta Q ; k:$ nombre entier, $\delta Q$ : débit passant entre deux lignes de courant successives que l'on trace. On trace en pratique un nombre de courbes orthogonales tel que les rectangles curvilignes obtenus soient en moyenne des carrés.

Pour obtenir la relation permettant le tracé on applique le théorème intégral de Stokes aux rectangles curvilignes obtenus :

$$
\left.\int_{A} \overrightarrow{(\operatorname{rot}} \vec{V}\right)_{n} d a=\int_{C} \mathrm{~V}_{\mathrm{S}} d s
$$

Sur la figure 3 , on voit que :

$$
\int_{\mathrm{A}}(\overrightarrow{\operatorname{rot}} \overrightarrow{\mathrm{V}})_{n} d a \# / \operatorname{rot} \mathrm{V}_{i} \cdot \frac{d_{1}+d_{3}}{2} \cdot\left(d_{2}+d_{4}\right)
$$

où /rot V/ est pris au centre du rectangle. 


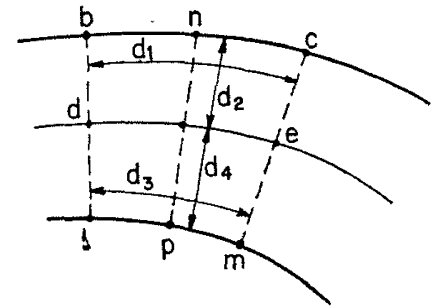

FIG. 3

$$
\begin{gathered}
\mathrm{V}_{s} \# \frac{\mathrm{Q}}{\mathrm{H}_{2} d_{2}} \text { sur } \ll b c » \\
\mathrm{~V}_{s} \# \frac{\mathrm{Q}}{\mathrm{H}_{4} d_{4}} \mathrm{sur} \ll \operatorname{lm} » \\
\mathrm{~V}_{s}=0 \text { sur } \ll b l » \text { et } \ll c m »
\end{gathered}
$$

où $\mathrm{H}_{2}$ et $\mathrm{H}_{4}$ sont les hauteurs de l'écoulement en $« \gg$ et $\ll p »$.

On en déduit :

$$
\left(\frac{d_{1}}{\mathrm{H}_{2} d_{2}}-\frac{d_{3}}{\mathrm{H}_{4} d_{4}}\right) \frac{1}{\left(\mathrm{H}_{2}+\mathrm{H}_{4}\right)\left(d_{1}+d_{2}\right)\left(d_{3}+d_{4}\right)}=\mathrm{F}(\mathrm{Q})
$$

Voyons dans un cas particulier comment on peut se servir de cette relation pour tracer un écoulement. Soit à tracer l'écoulement dans un convergent (fig. 4). Dans la partie « $a \gg$ de cet

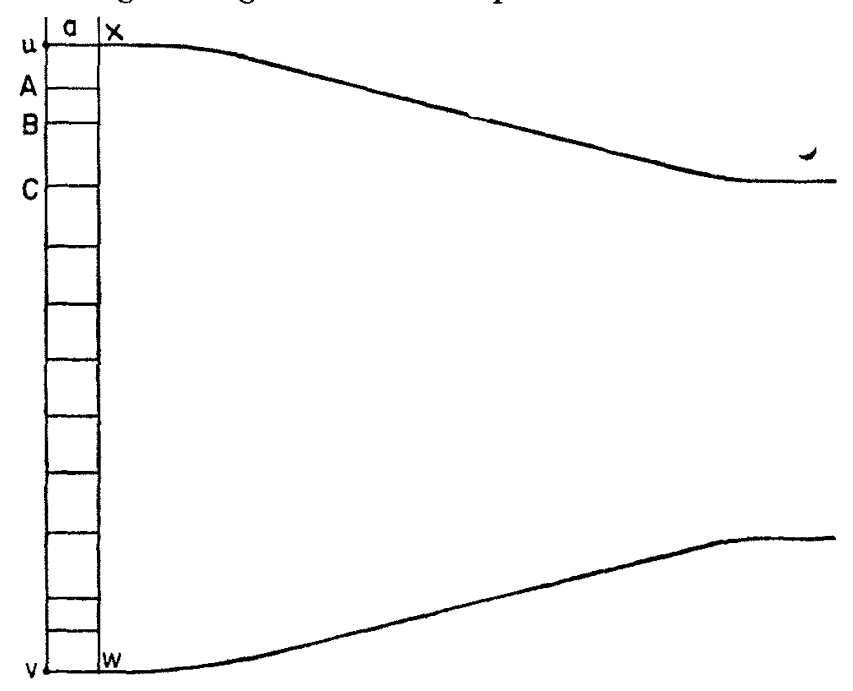

Fig. 4

écoulement, les vitesses sont par hypothèse parallèles et il faut s'y donner la répartition des débits le long du segment « $u v$ » à l'entrée du convergent. Si on connaît le débit le long de ce segment, on peut facilement trouver les points $A, B, C$ tels que les débits entre $A$ et $B, B$ et $C$, etc. soient les mêmes. Les points $\mathrm{A}, \mathrm{B}, \mathrm{C}$ sont donc les points de départ des lignes de courant que nous tracerons. Par hypothèse, dans la partie « $a »$, ces lignes de courant sont des parallèles.

$\mathrm{Si}$ on trace alors une ligne orthogonale quelconque, par exemple la ligne « $t w x »$, on peut calculer, le long de chaque ligne de courant, la grandeur : $F(Q)$. On connaîtra donc à partir de là une relation entre les longueurs de rectanbles curvilignes futurs le long de chaque ligne de courant.

Pour tracer l'écoulement, on procède d'une façon analogue aux tracés empiriques d'écoulement irrotationnels de fluide parfait par la méthode Lehmann. La méthode de tracé à employer dans notre cas est très voisine de la méthode de Lehmann, seule la relation entre les côtés des rectangles curvilignes est plus compliquée dans notre cas. D'ailleurs si, dans notre cas, le rotationnel est nul, on retrouve la relation valable pour le tracé de Lehmann. En effet, dans ce cas, la grandeur $\mathrm{F}(\mathrm{Q})$ est nulle et la relation précédente entre les longueurs des côtés des rectangles curvilignes devient $d_{1}=d_{3}$ si $d_{2}=d_{4}$ (si toutefois la hauteur de l'écoulement est constante, comme c'est le cas dans la méthode de Lehmann).

En outre, dans les deux cas, le tracé ne peut pas être fait du premier coup. En effet, comme nous l'avons vu, tous les points d'un écoulement s'influencent mutuellement les uns les autres; par conséquent, la condition imposée à l'écoulement à la sortie du convergent influe sur le tracé de l'écoulement en tous les points du convergent et ceci, aussi bien dans la méthode de Lehmann que dans la méthode actuelle. D'ailleurs, l'analogie va plus loin : la condition qu'on peut s'imposer à la sortie du convergent est la même dans les deux méthodes. Dans les deux cas, on ne peut s'imposer qu'une seule relation entre les deux composantes de la vitesse à la sortie.

Dans le cas actuel du convergent, cette relation traduit évidemment que la composante de la vitesse perpendiculaire à l'axe du convergent doit être nulle assez loin à l'aval du convergent. Ce n'est qu'à l'entrée que la condition imposée dans les deux cas est différente. Dans le cas des écoulements irrotationnels de fluide parfait, on ne peut s'imposer qu'une condition entre les deux composantes de la vitesse à l'entrée, mais dans le cas actuel, on peut s'imposer deux conditions entre les deux composantes, c'est-à-dire on peut s'imposer exactement la valeur de la vitesse en chaque point. C'est justement cette différence qui permet d'obtenir des écoulements beaucoup plus exacts dans le cas actuel, comme nous l'avons indiqué précédemment.

Du point de vue mathématique, les conditions à imposer à la fonction $Q$ aux limite's du champ d'intégration sont les mêmes pour les équations aux dérivées partielles : 
$\Delta Q=0$ et $\Delta Q=f(Q)$ si $f(Q)$ est fixé. Par contre, si $f(Q)$ n'est pas fixé, il faut évidemment se donner une condition de plus sur chaque ligne de courant dans le cas de l'équation $\Delta \mathrm{Q}=f(\mathbf{Q})$. Mathématiquement, il se pose un problème si on ne sait pas à priori où passent les lignes de courant. La figure 5 montre un exemple. Dans le cas $A$, il faut se donner une condition supplémentaire sur « $a b »$ et « $c d »$, dans le cas B seulement sur « $a b$ ». En pratique, une telle ambiguïté n'existe à peu près jamais.
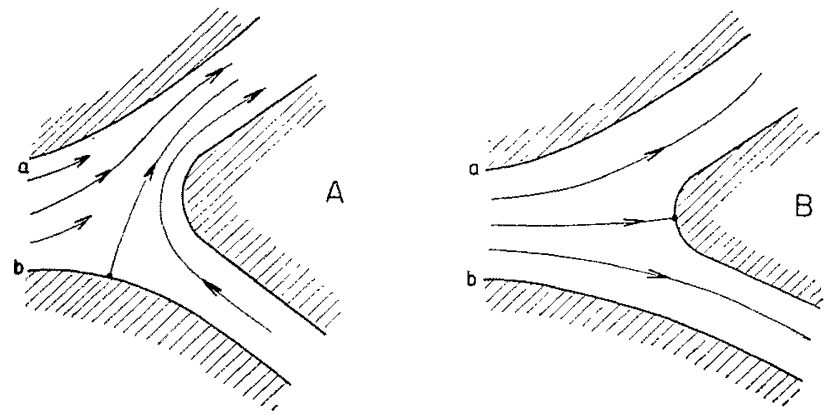

FIG. 5

La figure 6 montre le tracé complet de l'écoulement dans un convergent en admettant une répartition des vitesses de Nikuradze à l'entrée du convergent. La figure 7 montre l'allure des courbes de vitesses à l'entrée et à la sortie du convergent. On voit en particulier que les vitesses deviennent de plus en plus uniformes dans un convergent, résultat bien connu expérimenta-

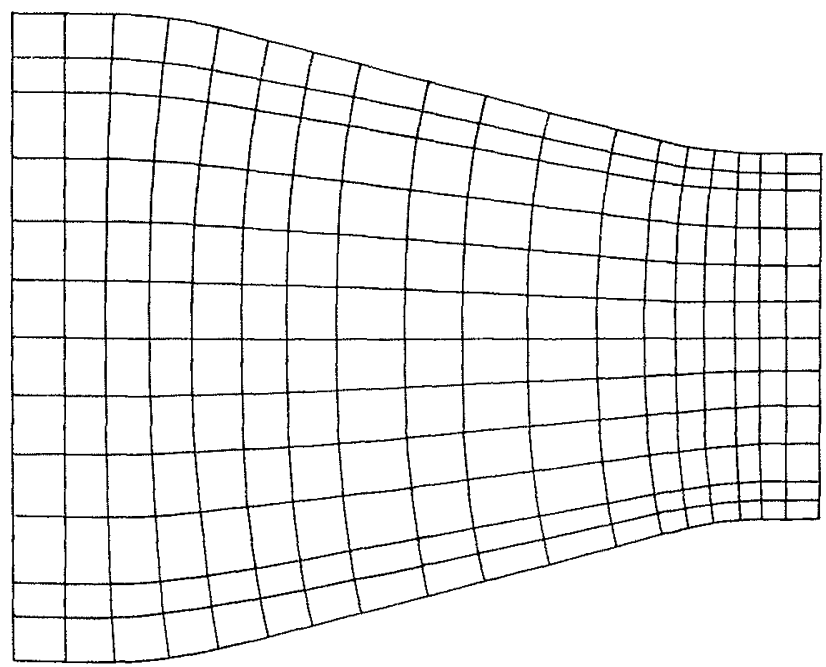

FII. 6

lement et que l'on retrouve ici par la méthode des écoulements rotat:onnels des fluides parfaits.

La figure 8 montre l'ćcoulement dans un divergent. Dans ce cas, on voit apparaitre un phénomène nouveau n'existant pas dans les écoulements irrotationnels. Ce phénomène est celui du décollement du fluide de la paroi au point où la vitesse tombe à zéro. Nous en reparlerons plus loin. Les zones hachurées sont des zones d'eau morte.

Les tracés d'écoulement de révolution se font à l'aide d'une formule du même type que celle

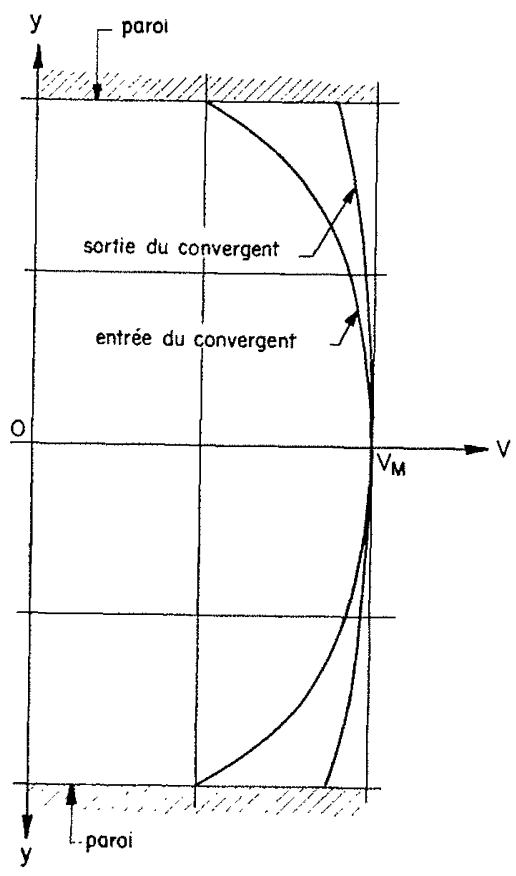

FIG. 7

valable pour les écoulements plans. En plus, dans ce cas, les difficultés de tracé sont à peu près les mêmes, que les écoulements soient rota-

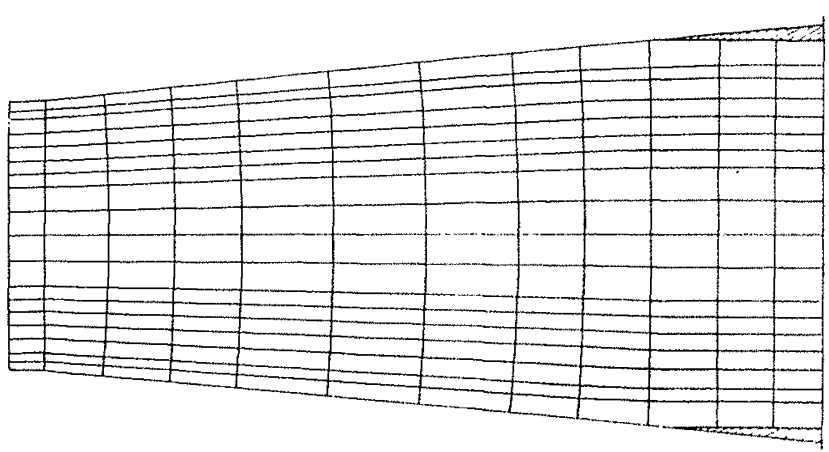

Fic. 8

tionnels ou irrotationnels. En effet, dans les deux cas, Ies deux familles de courbes à tracer forment entre elles des rectangles et il faut s'assurer, par un calcul, que les côtés de ces rectangles vérifient une certaine relation faisant intervenir entre autres la distance moyenne du rectangle à l'axe de révolution. De plus, les ćcoulements irrotalionnels de révolution ne peuvent être tracés 
qu'empiriquement, tout comme les écoulements rotationnels de révolution. En effet, la théorie des fonctions analytiques qui permet de calculer les écoulements irrotationnels plans, ne s'applique pas dans le cas des écoulements de révolution.

Insistons encore une fois sur le fait que l'on peut tracer aussi bien les écoulements méridiens que les écoulements dans lesquels il existe une composante de la vitesse des particules le long des cercles parallèles, c'est-à-dire les écoulements dans lesquels l'eau tourne en spirales autour de l'axe de révolution. Dans ce cas, on trouve, par le présent tracé, les intersections des surfaces de courant par un plan méridien. On peut facilement en déduire les vitesses méridiennes.
En plus, on a vu que l'intensité des vitesses le long des cercles parallèles sur une même surface de courant est donnée par la formule :

$$
\mathrm{V}_{p}=\frac{\mathrm{K}}{r}
$$

où $r$ est la distance du point considéré à l'axe de révolution. Oni peut donc calculer en tous les points les vitesses parallèles si on connaît ces vitesses dans la section d'entrée. Il existe évidemment aussi, dans le cas des écoulements de révolution, des surfaces de décollement analogues à celles décrites dans le cas des écoulements plans.

\section{VII. - QUELQUES PROPRIETTES NOUVELLES DE CES ECOULEMENTS DEDUITES DES TRACÉS GRAPHIQUES}

Parlons de quelques propriétés des écoulements rotationnels des fluides parfaits que l'on peut déduire des tracés graphiques.

Pour montrer, une fois de plus, l'intérêt pratique de ces tracés et pour ne pas trop allonger cette communication, nous nous contenterons d'examiner quelques propriétés relatives à un cas particulier important dans la pratique. Tout à l'heure, nous avons parlé des culottes, nous parlerons maintenant des aspirateurs en cône droit des turbines.

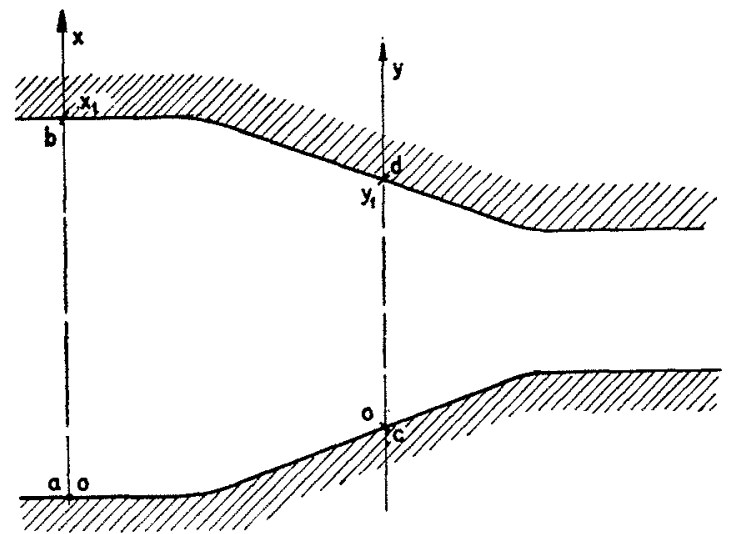

FIG. 9

Une première propriété importante que l'on peut constater à l'aide des tracés graphiques est qu'on peut calculer les écoulements convergents et divergents comme s'ils étaient formés d'une suite d'écoulements parallèles de largeur variable pourvu que la conicité reste inférieure à $15^{\circ}$ (angle total) et ne varie pas trop brusquement.
La forme des sections droites peut d'ailleurs varier.

Montrons comment on peut calculer un éconlement plan à filets parallèles : figure 9. Admettons que l'on connaisse la répartition des vitesses le long de l'axe $\mathrm{O} x: u=\boldsymbol{u}(x)$. On veut chercher la répartition des vitesses sur l'axe $\mathrm{O} y: v=v(y)$. Soit $y=y(x)$ la fonction qui fait correspondre les points de l'axe $\mathrm{O} y$ aux points de l'axe $O x$ qui sont sur une même ligne de courant. On a alors :

$u d x=v d y:$ conservation du débit,

et :

$\frac{d u}{d x}=\frac{d v}{d y}:$ conservation du rotationnel le long de chaque filet.

On peut calculer $d v$ à partir de chacune des deux relations ci-dessus. On obtient :

$$
d v=d\left(u \frac{d x}{d y}\right)=\frac{d u}{d x} d y
$$

Posons :

d'où :

$$
\frac{d x}{d y}=p
$$

$$
p d u+u d p=\frac{1}{p} d u
$$

On peut aussi écrire :

$$
\frac{d u}{u}=-\frac{1}{2} \frac{d\left(1-p^{2}\right)}{1-p^{2}}
$$


Done :

$$
\begin{gathered}
\frac{1}{\sqrt{1-p^{2}}}=\mathrm{A} u ; \mathrm{A}: \text { constante d'intégration, } \\
p=\frac{\sqrt{\mathrm{A}^{2} u^{2}-1}}{\mathrm{~A} u}=\frac{d x}{d y}
\end{gathered}
$$

$u(x)$ étant par hypothèse connue, on en déduit :

$$
y=\int_{0}^{\infty} \frac{u d x}{\sqrt{u^{2}+\mathrm{B}}}
$$

avec $\mathrm{B}=-\frac{1}{\mathrm{~A}^{2}}$ : constante d'intégration

La constante B est donnée par :

$$
y_{1}=\int_{0}^{x_{1}} \frac{u d x}{\sqrt{u^{2}+\mathrm{B}}}
$$

$x_{1}$ et $y_{1}$ étant les largeurs de l'écoulement sur les axes $\mathrm{O} x$ et $\mathrm{O} y$ (voir fig. 9).

D'autre part :

$$
\begin{gathered}
d y=\frac{u d x}{\sqrt{u^{2}+\mathrm{B}}} \\
\mathrm{el} \mathrm{:} \quad v=\frac{u d x}{d y}=\sqrt{u^{2}+\mathrm{B}}
\end{gathered}
$$

En pratique, on part d'une répartition $u(x)$ et d'une largeur $x_{1}$. Dès qu'on se fixe $B$ on peut calculer la nouvelle largeur $y_{1}$, la graduation des lignes de courants et les vitesses sur l'axe de largeur $y_{1}$ du convergent ou du divergent. Un calcul analogue peut être fait dans le cas des écoulements presque plans et méridiens de révolution; par contre, pour les écoulements plus compliqués, en particulier les écoulements de révolution non méridiens, le problème mène à une équation différentielle du deuxième ordre. La résolution est néanmoins beaucoup plus simple que la résolution de l'équation aux dérivées partielles du troisième ordre :

$$
\frac{\partial Q}{\partial x} \frac{\partial \Delta Q}{\partial y}=\frac{\partial Q}{\partial y} \frac{\partial \Delta Q}{\partial x}
$$

valable dans le cas général.

Le calcul ci-dessus permet aussi de trouver les décollements et leur raison. L'équation (5) montre que $y_{1}$ est une fonction décroissante de $B$, donc $B$ une fonction décroissante de $y_{1}$, et le maximum de $y$ est obtenu pour la plus petite valeur de $B$. Cette valeur est $-u_{m}{ }^{2}, u_{m}$ étant la vitesse $u(x)$ la plus petite. La largeur maximum du jet sortant d'un diffuseur est donc, dans le cas de la présente théorie :

$$
\eta_{n}=\int_{0}^{x_{1}} \frac{u d x}{\sqrt{u^{2}-u_{m}^{2}}}
$$

Si la largeur du divergent croît au-dessus de $y_{1}$, il y a nécessairement un décollement. Les décollements limitent la possibilité de récupération d'énergie cinétique par les divergents. Dans le cas d'un divergent plan, l'énergie cinétique minimum sortant du divergent est :

$$
\frac{1}{2} \int_{0}^{\nu_{m}} v^{2} d y=\int_{0}^{x_{1}} \sqrt{u^{2}-u_{m}^{2}} u d x
$$

Dans le cas des aspirateurs en cône droit des turbines, l'écoulement est de révolution et non méridien. Dans ce cas, il y a en général deux possibilités de décollement: au centre et près de la paroi. Ces décollements dépendent évidemment (comme dans le cas des divergents plans) de la répartition des vitesses à l'entrée du divergent, donc à la sortie de la turbine. On voit donc que l'énergie maximum récupérable par un diffuseur en cône droit dépend d'une part de la turbine et d'autre part de la façon d'alimenter cette turbine, puisque la répartition des vitesses à la sortie de la turbine dépend de la répartition à l'entrée.

Comme, dans les basses chutes, la récupération de l'énergie par l'aspirateur peut dépasser $50 \%$ de l'énergie totale, on voit que pour faire des essais valables sur modèle réduit, il faut en particulier représenter exactement la courbe de répartition des vitesses à l'entrée de la bâche. D'ailleurs le problème de la récupération de l'énergie par les aspirateurs est de toute façon très complexe; en effet, selon le régime de fonctionnement (puissance débitée et chute), la répartition des vitesses à la sortie des roues varie fortement, aussi bien la répartition des vitesses méridiennes que la répartition des vitesses suivant les cercles parallèles.

En plus, la viscosité finit par agir quand les divergents sont longs. Si l'angle au sommet du cône était très petit, l'écoulement serait encore dans chaque section voisin d'un écoulement à filets parallèles; mais cet écoulement serait l'écoulement ordinaire dans une conduite, compte tenu de la rugosité. Si la rugosité absolue est constante dans un divergent, la rugosité relative diminue le long de l'écoulement et la courbe des vitesses deviendrait de plus en plus plus plate.

En fail, la rugosité intervient bien en pratique, mais les angles an sommet des cônes sont en génćral tels que seule une couche située près de la paroi est influencée. I'influence des parois décroît le long de l'écoulement. Comme la viscosité continue à détruire les tourbillons au sein du fluide, le rotationnel décroît dans la couche de fluide située près de la paroi, contrairement à l'hypothèse de base de la théorie des fluides parfaits.

Le rotationnel fixe la pente de la tangente à la courbe des vitesses $(d u / d x)$, donc la courbe 
devient plus plate : la viscosité empêche, ou du moins retarde les décollements dans les divergents classiques. Elle augmente évidemment aussi les pertes de charge, mais dans l'ensemble le rendement augmente très nettement par rapport à la théorie des écoulements rotationnels des fluides parfaits. On voit ainsi l'importance d'une rugosité aussi faible que possible pour le rendement des aspirateurs en cône droit industriels.

Finalement, on voit que la théorie des écoulements rotationnels des fluides parfaits est essentiellement limitée par l'intervention de la viscosité. En effet, en dehors de l'absence de la viscosité dans une partie de l'écoulement, au- cune autre hypothèse simplificatrice n'est plus faite. Les hydrauliciens se contenteraient très souvent de connaître la variation du rotationnel moyen le long des filets fluides, c'est-à-dire une relation plus exacte que la constance du rotationnel le long des filets (en écoulement plan), mais il est clair que le rotationnel ne peut pas être étudié tout seul en tant que tel : en effet, les tourbillons sont précisément entraînés le long des filets; il faudrait donc connaître la vitesse avant de calculer le rotationnel. Par ailleurs, il ne semble pas possible d'isoler le rotationnel en moyenne de ce qui se passe à petite échelle, et là on soulève tout le problème de la turbulence.

\section{VIII. - CONCLUSIONS}

Les conclusions de notre exposé sont les suivantes :

$1^{\circ}$ Nous avons montré que les écoulements rotationnels des fluides parfaits schématisent assez bien un grand nombre d'écoulements importants, surtout en hydroélectricité. Cette schématisation est, d'une part, nettement meilleure que celle qui consiste à admettre les écoulements irrotationnels; d'autre part, elle possède ellemême des limites dont nous venons de mentionner les principales;

$2^{\circ}$ Nous avons montré comment on peut tracer et étudier ces écoulements, en particulier dans les cas où ils peuvent être ramenés à deux dimensions. Parmi les écoulements que l'on peut ramener à deux dimensions, figurent en particulier les écoulements presque plans' et les écoulements de révolution méridiens ou non;

$3^{\circ}$ Nous avons montré comment on pouvait, dans certains cas, calculer ces écoulements;

4" Nous avons montré qu'il existe des décollements dus à la divergence des écoulements, même si on admet que le fluide est parfait. Nous avons aussi montré pourquoi ces décollements peuvent être évités si les angles au sommet des divergents sont suffisamment petits. La viscosité joue un rôle favorable, elle détruit les tourbillons dans la masse du fluide; la rugosité joue un rôle défavorable, elle crée des tourbillons près des parois;

$5^{\circ}$ Pour étudier plus exaclement les écoulements, il faut faire intervenir la viscosité.

Au passage, nous avons défini les écoulements presque plans qui ont, à notre avis, une importance technique très grande. En effet, les écoulements rigoureusement plans sont extrêmement rares, de même d'ailleurs que les écoulements de révolution rigoureusement méridiens.

Les écoulements presque plans, plans et de révolution peuvent être tracés (et même, dans certains cas, calculés) de facon relativement simple par une méthode analogue et guère plus compliquée que les écoulements: irrotationnels tracés empiriquement, qui ont été les seuls pratiquement connus jusqu'à présent et qui schématisent, dans beaucoup de cas, très mal les écoulements réels.

\section{I S C U S S I O N}

président : M. Remenieras

M. le Président remercie M. Meyer et souligne l'intérêt
des écoulements dits « rotationnels en fluides parfaits 》
en vue de certaines applications pratiques (aux diffuseurs
de turbines par exemple).
M. Bonnis rappelle que l'aplatissement de la courbe de distributions des vitesses dans un convergent - et l'évolution inverse dans un divergent - est plus qu'un fait d'expérience et peut être démontré qualitativement à partir du théorème de Bernoulli et même en première approximation, quantitativement. 
M. Meyer précise que dàns son exposé il s’agissait de tracer exactement les lignes de courant, ce que ne permet pas le théorème de Bernoulli.

M. Ie Président pense qu'il serait intéressant de présenter une application numérique de ces formules à un cas particulier.

Lc dernier cliché projeté représentait un diffuseur et M. Darrieus s'étonne de ce que les lignes de courant se resserraient près de la paroi pour s'écarter ensuite.

Ce paradoxe apparent est, précise M. MEyer, un effet d'optique dû au fait qu'il a fallu serrer les lignes là où il y avait une grosse variation du rotationnel dans l'écoulement. Les orthogonales n'ont aucune signification ef n'existent uniquement que pour faciliter le tracé et sa vérification de même que dans la méthode de Lehmann.

M. Darrieus pense que le tracé des rectangles doit vérifier une relation équivalente à la conservation du débit.

M. Meyer précise que, d'après le théorème de Stokes, la formule de base du tracé fait intervenir non seulement les longueurs des côtés des rectangles curvilignes tracés, mais aussi la valeur du rotationnel qui reste constante le long de chaque filet fluide dans le cas des écoulements plans et qui se déduit des conditions aux limites. Voir in fine les précisions données par M. DARRieus après la séance.

M. Escande remarque que s'il est vrai, ainsi que l'a fait remarquer M. Meyen lors de la projection de profils d'ailes, qu'en hydraulique la vitesse, à la paroi, est nulle contrairement à la théorie des fluides parfaits, il n'en est pas de même au bord de la couche limite, ce qui permet, en remplaçant la paroi de l'aile par le bord de la couche limite, de retrouver la théorie des fluides parfaits. C'est pourquoi on obtient avec cette théorie un degré d'approximation convenable.

De plus, il a été dit que dans une aile on ne pouvait pas expliquer le phénornène de circulation. D'après le théorème de Lagrange, un tourbillon ne peut naitre spontanément dans un fluide parfait tant que les forces massiques dérivent d'un potentiel. Mais inversement, r'après les théorèmes d'Helmholtz, un tourbillon existant à un instant donné se perpétue indéfiniment et ne peut disparâtre : il demeure constitué des mêmes particules fluides et son intensité reste inchangée.

Le phénomène de circulation autour de l'aile peut donc s'expliquer sans difficulté à partir du moment où l'on admet l'existence du tourbillon lié à l'aile.

M. MEYer remercie M. Escande des précisions qu'il a apportées à son exposé qui ne fut pas assez nuancé à cause du manque de temps. En effet, la théorie des fluides parfaits ne doit être nullement dénigrée. En étudiant de plus près la question, on s'apercoit d'ailleurs que la théorie des écoulements rotationnels des fluides parfaits ne peut servir que très peu en aérodynamique parce qu'on ne peut pas diviser les écoulements en plusieurs parties à l'entrée desquelles on connait le rotationnel. Comme nous l'avons vu, cette condition est nécessaire pour pouvoir appliquer à une partie d'un écoulement la théorie des écoulements rotationnels des fluides parfaits.

Par contre, comme nous l'avons déjà signalé, cette théorie s'applique très souvent en hydraulique où on connaît souvent la répartition des vitesses à la sortie des conduites et des canaux qui sont en même temps l'entrée d'organes tels que les culottes, les bâches, les divergents, etc., organes qui ont précisément été étudiés plus spécialement ici.

M. Hupner remarque que M. Meyer a beaucoup insisté sur l'intérêt qu'il y a à bien profiler les culottes dans les installations hydroélectriques et s'étonne qu'une industric aussi répandue que celle du chauffage central à eau chaude paraisse ignorer complètement ce principe et fasse encore des branchements à angle droit, ce qui peut sans doute expliquer le mauvais fonctionnement de bien des radiateurs. Il suggère l'ètude de ces phénomènes qui, dans les installations de chauffage central, sont encore compliquées par les différences de température.

M. le Président précise qu'aux U.S.A. « l'hydrauliquc des installations sanitaires et domestiques $\gg$ est très développée et a fait l'objet de nombreux essais de laboratoire. Tandis qu'en France, ainsi que le fait remarquer M. Mexer, pour le chauffage des hopitaux, par exemple, où l'on impose des minima et des maxima de température, les installateurs sont souvent très gênés pour satisfaire les cahiers des charges, parce que la technique de ces écoulements n'est pas eitcore bien connue en France.

Après la séance, M. DARnieus nous a adressé le développement ci-après de son intervention :

Le tracé graphique des écoulements permanents rotationnels avec conservation du tourbillon peut s'obtenir par une généralisation de la méthode de Lehmann, en considérant l'écoulement comme une juxtaposition de filets de courant chacun irrotationnel et séparés les uns des autres par des nappes tourbillonnaires le long desquelles la conservation, en vertu du théorème de Bernoulli, de l'écart $\left(v^{2}-v^{\prime 2}\right) / 2$ du demi-carré des vitesses de part et d'autre de la nappe de discontinuité au

$$
\left(v-v^{\prime}\right)\left[\left(v+v^{\prime}\right) / 2\right]=\mathrm{C}^{\mathrm{te}},
$$

cxprime la conservation, le long de la ligne de séparation, du débit de l'intensité tourbillonnaire $\left(v-v^{\prime}\right)$ entraînée avec la vitesse moyenne $\left(v+v^{\prime}\right) / 2$.

Or, si $d$ et $d^{\prime}$ représentent les écartements des écuipotentielles qui correspondent à un même échelón de potentiel, de part et d'autre de la ligne de séparation, les vitesses sont proportionnelles à $d^{-1}$ et $d^{\prime-1}$, de sorte que le tracé par tâtonnements devra être conduit en assurant, non plus la continuité des équipotentielles à travers tout le champ, mais des discontinuités telles que les quantités $1 / d^{2}-1 / d^{\prime 2}$ se conservent le long des lignes de courant.

Nous avons utilisé cette méthode il $y$ a quelques années pour la détermination, dans les tuyères de mesure de débits de fluides, du profil approché de vitesse au col qui correspond à un profil initial arbitrairement donné en travers de la conduite amont.

\section{杂.}

De son côté, M. Meyen nous a adressé les lignes suivantes :

C'est avec un très grand plaisir que nous avons pris connaissance de la lettre que nous a fait l'honneur d'écrire M. Darnieus. En effet, un problème important se pose à notre avis en matière d'écoulement de l'cau dans la technique des constructions « a grand nombre de Reynolds 》 (hydroélectricité, chauffage, etc.). Il s'agit de convaincre les hydrauliciens que les études théoriques restent possibles, malgré l'échec pratique de la théorie des écoulements irrotationnels dans de nombrcux domaines. Pour cela, il faut montrer que la théoric des écoulements rotationnels constitue un moyen d'étude valable à l'échelle de l'ingénieur, valable parce que suffisamment précis, suffisamment aisé à mettre en ouvre et. très riche en enseignements pratiques, comme nous avons essayé de le montrer dans notre communication. C'est pourquoi nous remercions très vivement $M$. 1)AnRIEUs pour les témoignages qu'apporte à notre méthode l'application de celle-ci aux tuyères de mesure. 